\title{
3D MESH SEGMENTATION USING LOCAL GEOMETRY
}

\author{
Sumanta Guha \\ Computer Science \& Information Management Program, Asian Institute of Technology, Thailand.
}

\begin{abstract}
.
We develop an algorithm to segment a $3 D$ surface mesh intovisually meaningful regions based on analyzing the local geometry ofvertices. We begin with a novel characterization of mesh vertices as convex,concave or hyperbolic depending upon their discrete local geometry.Hyperbolic and concave vertices are considered potential feature regionboundaries. We propose a new region growing technique starting fromthese boundary vertices leading to a segmentation of the surface thatis subsequently simplified by a region-merging method. Experiments indicatethat our algorithm segments a broad range of $3 D$ models at aquality comparable to existing algorithms. Its use of methods that belongnaturally to discretized surfaces and ease of implementation makeit an appealing alternative in various applications.
\end{abstract}

\section{KEYWORDS:}

Feature extraction, hyperbolic vertex, local geometry, meshsegmentation, region growing, region merging

\section{INTRODUCTION}

Polygonal meshes are the dominant mode of representing surfaces in computer graphics. They are geometrically simple and intuitive, and lend themselves to efficient algorithms and data structures. However, other than vertex-edge-face adjacency data, a polygonal mesh does not intrinsically possess any high-level semantic structure. For example, there is no information as such in the mesh data of the hand in Figure 1 that distinguishes the five fingers. However, such segmentation of an object into perceptually meaningful regions is important in applications such as shape recognition [22], morphing [8], texturing [11] and collision detection [12], amongst others.

The goal of surface mesh segmentation then is to decompose the input mesh into smaller regions which are perceptually significant. Nevertheless, as Atteneet al [1] point out it is not possible to define exactly what constitutes perceptual significance. Not only does this lie "in the eyes of the beholder" it may vary from application to application. The viewer's world knowledge is critical to segmentation as well - in a model that a layman would segment only into heart and lung and tissue, an oncologist may distinguish cancerous tumors and benign growths as well. 


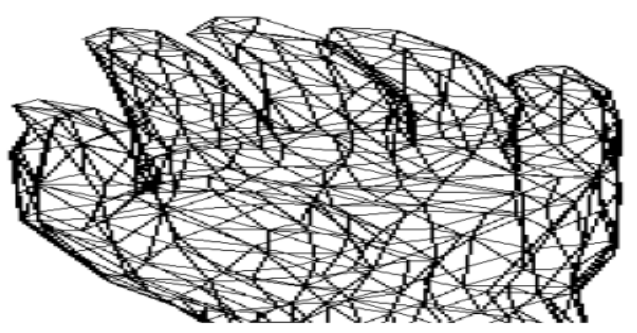

Fig. 1. Hand model.

Our contribution is a novel approach to mesh segmentation that first separates vertices based on their local geometry. In particular, we distinguish between convex, concave and hyperbolic vertices by examining their geometric neighbor- hoods as a discrete structure. This characterization, which seems to be new in CG applications, is not derived from the well-known method of differentiating between hyperbolic, parabolic and elliptic points on a smooth surface using Gaussian curvature, though the two are not unrelated. Following the characterization of vertices, we estimate the potential (meaningful) region boundaries to be along the concave and hyperbolic vertices. Regions are subsequently grown from the boundary vertices by a novel modification of the watershed segmentation scheme. A final region merging step bounds the number of segments.

The rest of the paper is organized as follows. Section 2 briefly reviews existing algorithms that are relevant to ours. In Section 3 we discuss the background theory and our segmentation method. Section 4 shows experimental results on various 3D models and Section 5 concludes the paper.

\section{RELATED WORKS}

An approach to segmentation that splits the surface into coherent nearly-flat patches based, typically, on a curvature analysis is called patch-type segmentation [7, 5, 19, 22]. See Figure 2. Patch-type segmentation is used in applications that are sensitive to geometric properties such as planarity and convexity. These include texture mapping, re-meshing and simplification [18].
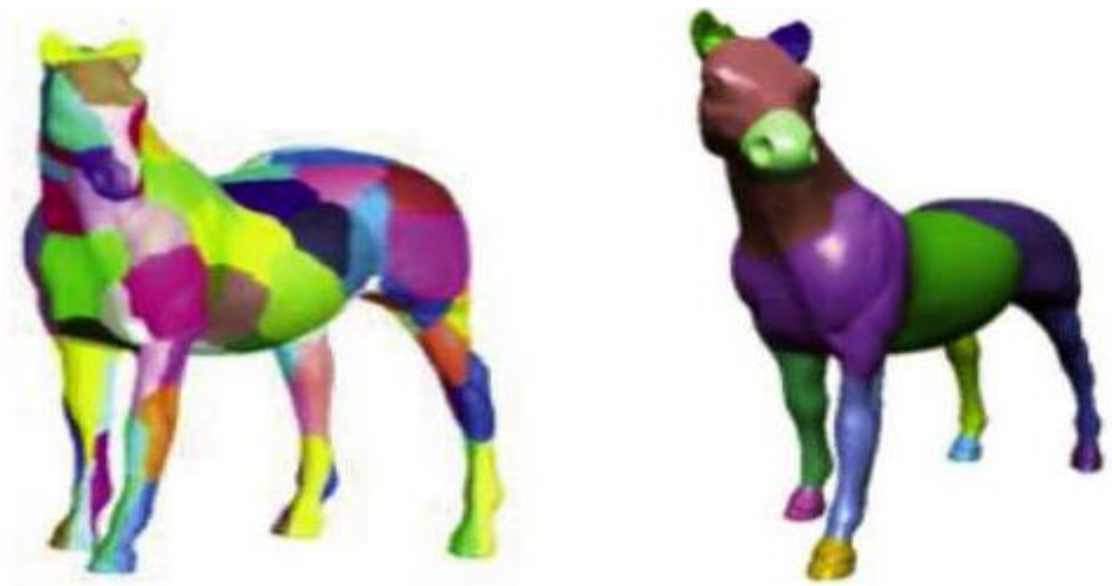
International Journal of Computer Graphics \& Animation (IJCGA) Vol.5, No.2, April 2015

Fig. 2. Patch-type (left) and part-type (right) segmentation taken from [18].

Part-type segmentation, on the other hand, seeks to sub-divide the input mesh into sub-meshes that match human perception. Algorithms of this type can assist applications such as shape retrieval, shape recognition, collision detection and object manipulation. Rom and Medioni [16] in 1994 proposed a framework to decompose 3D objects into parts using the curvature properties of parabolic curves. Though they initiate the use of curvature in segmentation their approach cannot directly process triangle meshes.

Sacchi et al [17] and Mangan and Whitaker [14] propose approaches to seg- mentation using curvature that can apply to a mesh object. They use total curvature as the key to segmenting parts from an input mesh. However, their segmentation of large models often fails to match viewer perception.

Page et al [15] in 2003 introduced a method called Fast Marching Water- sheds that splits a triangle mesh into segments that fit the definition of visual parts according to the minima rule proposed by Hoffman and Richards [9]. The minima rule is an elegant theory from computer vision which propounds that human perception decomposes a 3D object into visual constituents at contours of negative Gaussian curvature - bands of hyperbolic points in other words.Some recent segmentation methods incorporate semantics derived, typically, from knowledge embodied in a training set: Kalogerakis et al [10] and Lv et al [13] simultaneously segment and label parts of a 3D mesh.

Zhang et al [21] proposed a segmentation algorithm that estimates the Gaussian curvature at a vertex, identifies the hyperbolic vertices as potential region.boundaries, applies the watershed technique to grow regions, and completes finally with a curvature-driven region merging phase. Recently, Chen and Geor-ganas [3] improved the algorithm of Zhang et al. [21] by extending the boundary detection step to additionally identify concave vertices and prevent them from breaking segment integrity.

Our segmentation algorithm is inspired by that of Chen and Georganas. How- ever, we do not invoke Gaussian curvature. Instead, we apply our new classification scheme to distinguish concave and hyperbolic vertices, which subsequently serve as region boundaries. Our region growing algorithm is a modification of the watershed technique that seems to better allow growth into intuitively separate parts.

\section{THEORY AND ALGORITHM}

\subsection{Vertex classification}

To simplify the theoretical development we assume that the input mesh is a topologically closed surface, implying that it has a meaningful interior. This as- sumption is not essential, however, and our code works for non-closed meshes as well. We assume that mesh faces are all triangular. We begin with a characterization of vertices as convex, concave or hyperbolic.

Definition 1. A vertex $\mathrm{V}$ of a mesh $\mathrm{M}$ is hyperbolic if it is contained in the interior (as a subspace of R3) of the convex hull $\mathrm{C}$ of its neighbors. A vertex $\mathrm{V}$ that is not hyperbolic is convex if there 
International Journal of Computer Graphics \& Animation (IJCGA) Vol.5, No.2, April 2015

exists a (flat) disc $\mathrm{D}$ centered at $\mathrm{V}$ which does not intersect the interior of $\mathrm{M}$; otherwise, it is concave.

For example, the convex hull of the neighbors of $\mathrm{V}$ in Figure 3(a) is the triangle W1W2W3, which has empty interior in $\mathrm{R} 3$. Therefore, $\mathrm{V}$ is trivially non- hyperbolic; moreover, the disc $\mathrm{D}$ proves that $\mathrm{V}$ is convex. The vertex $\mathrm{V}$ on the saddle-shaped surface in Figure 3(b) is hyperbolic. The reader may check in Figure 3(c) that all the vertices on the L-shaped solid are nonhyperbolic, and that only vertex $\mathrm{V}$ is concave, while all the rest are convex. The disc $\mathrm{D}$ at a corner of the L-shaped solid indicates the reason why we cannot replace the disc in the definition of convexity with its containing plane - a plane may intersect the mesh at a distant point.

Our characterization of a mesh vertex is not unrelated to that of a point of a smooth surface as hyperbolic, parabolic or elliptic by means of Gaussian curvature, but we'll not explore the connection here. It should be observed though
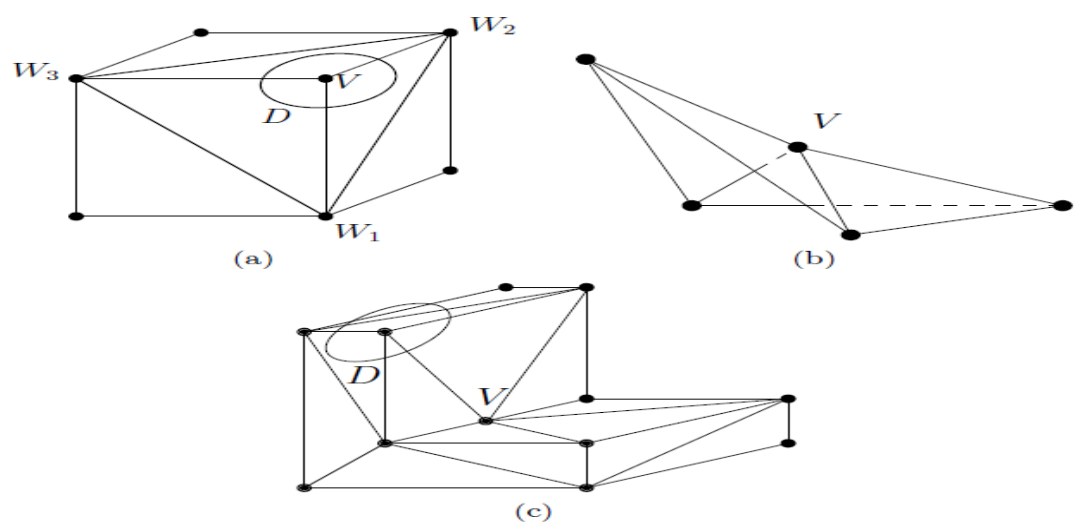

Fig. 3. Illustrations of convex, hyperbolic and concave vertices.

that our use of the discrete local geometry to classify vertices seems more natural for a meshed surface than computing pseudo-curvature values, as some authors do, on a smooth approximation.

Whether a vertex $\mathrm{V}$ is hyperbolic or not is a local decision depending on its disposition with respect to its neighbors. However, distinguishing a non- hyperbolic vertex $\mathrm{V}$ as either convex or concave necessitates one to determine the side of the surface near $\mathrm{V}$ that the interior of $\mathrm{M}$ lies, which requires global knowledge of $\mathrm{M}$.

\subsection{Region Growing}

Our strategy to find features is to first presume hyperbolic and concave vertices as potential feature region boundaries. The motivation is simple. A surface whose vertices are all convex, e.g., the box of Figure 3(a), is likely featureless. On the other hand, the two legs of the L-surface of Figure 3(c) appear to be features that are separated by the edge at the crook between them - all vertices in the interior of this edge being concave. Vertices along the circular region where the stem meets the cap of the mushroom of Figure 4 appear to be hyperbolic. 
The next stage in our strategy is, therefore, to treat the hyperbolic and concave vertices as"seeds", from which to grow the feature regions. In particular, we shall grow the regions in a scattershot manner as the local geometry at a seed

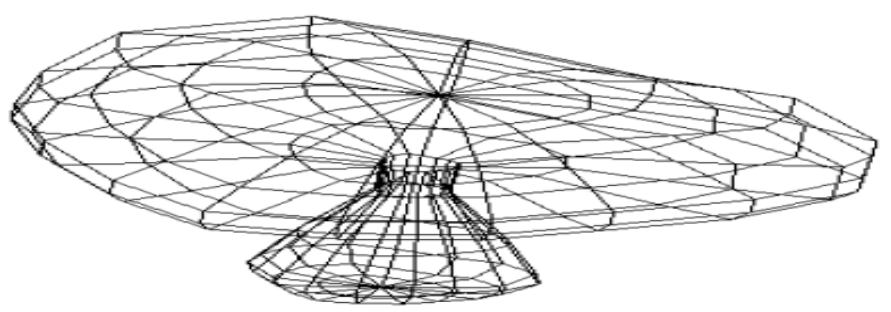

Fig. 4. Mushroom model.

does not in general suggest a best direction. The plan is as follows. Seeds are initially all labeled differently. They are next processed iteratively to label their neighbors. Vertices adjacent to each seed, which have not already been labeled, are given new and different labels. In the following rounds vertices already labeled are processed iteratively to propagate their labels in a breadth-first manner.

For example, say the labels after the first round of the vertices $w 1, w 2, \ldots$,wk, adjacent to a seed $\mathrm{v}$ are $11,12, \ldots, 1 \mathrm{k}$, respectively. Then, vertices adjacent to $\mathrm{w} 1$, that have not already been labeled, are labeled 11; then, those adjacent to w2, that have not already been labeled, are labeled 12; and so on. Figure 5 illustrates the idea. The process of growing feature regions in this manner from each seed is repeated iteratively until all the vertices of the mesh have been labeled.

Our region growing method, though inspired by the watershed technique, is different in that the latter labels regions with the names of the seeds themselves, while our method adds one layer of growth in every possible direction. This idea suggested itself from experimentation with various models and seems to actually work better than the straight watershed scheme in practice.

\subsection{Region Merging}

Vertices having like labels form a feature region, but there is likely great over-segmentation at this point, so we begin a third stage of region merging. In thisfinal stage, the feature region with the smallest number of vertices is iterativelymerged with a neighboring region. We choose the neighboring region to mergewith as the one - borrowing a heuristic from Chen and Georganas [3] - that

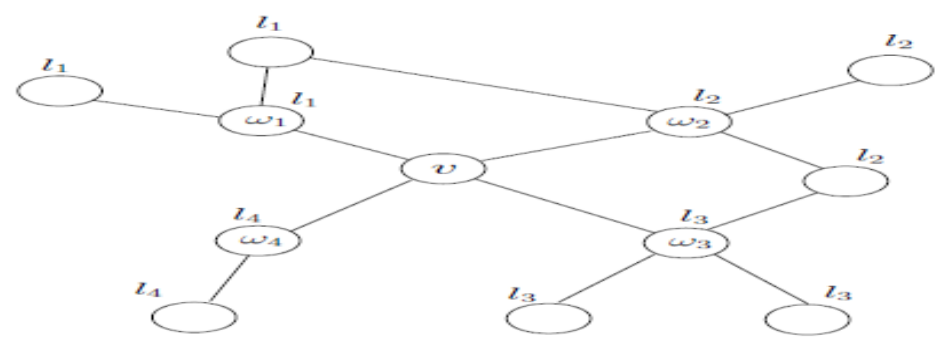


Fig. 5. Region growing.

shares the longest border with the candidate for merging. Figure 6 shows the idea. Region merging continues until a preset threshold is reached in the number of segments.
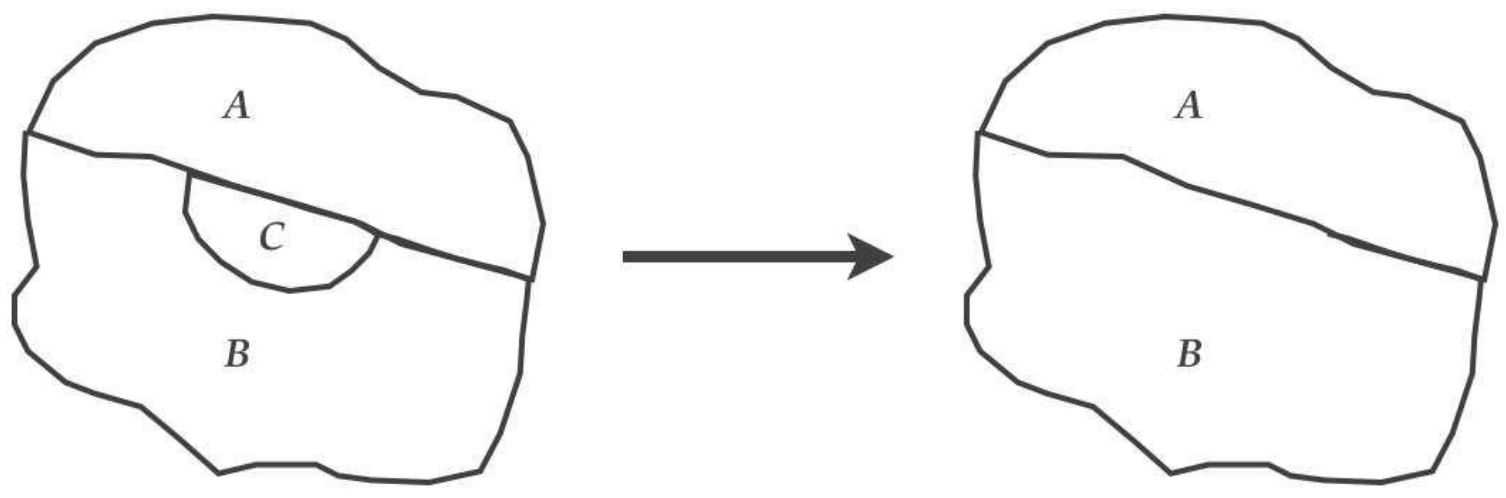

Fig. 6. Region merging.

\subsection{Complexity}

It is still fairly straightforward to see that the complexity of our segmentation procedure is linear in the size of the mesh, because: (a) the classification of vertices as convex/concave/hyperbolic requires examination only of each vertex's link, and, moreover, each edge of a surface mesh can appear in the link of at most two vertices, for a total cost linear in the number of edges; (b) region growing is a breadth-first procedure requiring time linear in the number of vertices; (c) region merging costs $\mathrm{O}(1)$ at most per vertex for a total linear cost as well.

\section{EXPERIMENTAL RESULTS}

We have implemented our segmentation algorithm, coding it in C++ with the STL on an Intel platform with $2 \mathrm{Ghz}$ CPU and 1GB RAM. We used several pre- packaged routines from the CGAL library [2], the Qt toolkit [20] to build the GUI, and the libQGLViewer [6] as a rendering engine. Figures 7-11 show experimental results on fairly large meshes. The quality of the output compares well with that of existing methods.

\section{CONCLUSIONS AND FUTURE WORK}

We have proposed a novel algorithm for segmenting meshed surfaces into smaller visually meaningful parts. We apply a discrete and local characterization, itself new, of vertices intohyperbolic, convex and concave, to find region boundaries. This method seems a more natural fit for a mesh structure than the popu-lar Gaussian curvature estimation method, the latter being a transplant fromsmooth surfaces. Our method of region growing is a novel modification as wellof the watershed method. 
International Journal of Computer Graphics \& Animation (IJCGA) Vol.5, No.2, April 2015

There are specific improvements that might be made. For example, the re-gion growing method is not robust and is sensitive to the initial labeling of theboundary vertices. A way to address this problem may be to "invert" the la-beling procedure so that it starts from the non-boundary vertices, each suchfinding a nearest (according to an appropriate distance metric) boundary vertex and adopting the latter's label. Additionally, it would be useful to be ableto replace the userset threshold for the number of regions at which the merg-ing process stops with automatictermination based upon some measure of thequality of the current segmentation.

Another important direction for future work is to extend the algorithm toprocess point cloud data, where there is no connectivity information.

\section{ACKNOWLEDGEMENTS}

We would like to thank Chansophea Chuon for helping develop our software andcollaborating on preliminary results announced in Chuon and Guha [4]. We aregrateful to the AIM@SHAPE Project for the mesh models that we used. Wethank Nguyen Tan Khoa for initial contributions.

\section{REFERENCES}

[1] M. Attene, S. Katz, M. Mortara, G. Patane, M. Spagnuolo, and A. Tal. Meshsegmentation - a comparative study. In SMI '06: Proceedings of the IEEE Inter-national Conference on Shape Modeling and Applications 2006 (SMI'06), page 7, Washington, DC, USA, 2006. IEEE Computer Society.

[2] http://www.cgal.org/.

[3] L. Chen and N. D. Georganas. An efficient and robust algorithm for 3d meshsegmentation. Springer Science + Business Media, LLC, 2006.

[4] C. Chuon and S. Guha. Surface mesh segmentation using local geometry. In Pro-ceedings of the 6th International Conference on Computer Graphics, Imaging andVisualization (CGIV '09), pages 250254, Tianjin, China, 2009. IEEE ComputerSociety.

[5] D. Cohen-Steiner, P. Alliez, and M. Desbrun. Variational shape approximation. InSIGGRAPH '04: ACM SIGGRAPH 2004 Papers, pages 905-914, New York, NY,USA, 2004. ACM Press.

[6] G. Debunnes. http://artis.imag.fr ${ }^{\sim}$ Gilles.Debunne/QGLViewer/.

[7] M. Eck, T. DeRose, T. Duchamp, H. Hoppe, M. Lounsbery, and W. Stuetzle.Multiresolution analysis of arbitrary meshes. In SIGGRAPH '95: Proceedings ofthe 22nd annual conference on Computer graphics and interactive techniques, pages173-182, New York, NY, USA, 1995. ACM Press.

[8] A. D. Gregory, A. State, M. C. Lin, D. Manocha, and M. A. Livingston. Interactivesurface decomposition for polyhedral morphing. The Visual Computer, 15(9):453-470, 1999.

[9] D. D. Hoffman and W. Richards. Parts of recognition. Technical Report AIM-732,Massachusetts Institute of Technology - Artificial Intelligence Laboratory, 1983.

[10] E. Kalogerakis, A. Hertzmann, and K. Singh. Learning 3d mesh segmentation andlabeling. In ACM Transactions on Graphics - Proceedings of ACM SIGGRAPH2010. ACM, 2010.

[11] B. Levy, S. Petitjean, N. Ray, and J. Maillot. Least squares conformal maps forautomatic texture atlas generation. ACM Trans. Graph., 21(3):362-371, 2002.

[12] X. Li, T. W. Toon, and Z. Huang. Decomposing polygon meshes for interactive applications. In I3D '01: Proceedings of the 2001 symposium on Interactive 3D graphics, pages 35-42, New York, NY, USA, 2001. ACM Press.

[13] J. Lv, X. Chen, J. Huang, and H. Bao. Semi-supervised mesh segmentation andlabeling. In Computer Graphics Forum - Proceedings of Pacific Graphics 2012,pages 2241-2246. ACM, 2012. 
International Journal of Computer Graphics \& Animation (IJCGA) Vol.5, No.2, April 2015

[14] A. P. Mangan and R. T. Whitaker. Partitioning 3d surface meshes using watershed segmentation. IEEE Transactions on Visualization and Computer Graphics, 5(4):308-321, 1999.

[15] D. L. Page, A. F. Koschan, and M. A. Abidi. Perception-based 3d triangle meshsegmentation using fast marching perception-based $3 \mathrm{~d}$ triangle mesh segmentationusing fast marching watersheds. Proceedings of International Conference onComputer Vision and Pattern Recognition, II:27-32, June 2003.

[16] H. Rom and G. Medioni. Part decomposition and description of 3d shapes. InPattern Recognition, pages 629-632, 1994.

[17] R. Sacchi, J. F. Poliakoff, P. D. Thomas, and K. H. Hafele. Curvature estimationfor segmentation of triangulated surfaces. In Digital Imageing and Modeling, pages536-543, 1999.

[18] A. Shamir. A formulation of boundary mesh segmentation. In 3DPVT '04: Pro-ceedings of the 3D Data Processing, Visualization, and Transmission, 2nd Inter-national Symposium on (3DPVT'04), pages 82-89, Washington, DC, USA, 2004.IEEE Computer Society.

[19] A. Sheffer. Model simplification for meshing using face clustering. In ComputerAided Desgin, pages 925-934, 2001.

[20] TrollTech. Qt 4.2.2 community version http://www.trolltech.com.

[21] Y. Zhang, J. Paik, A. Koschan, M. A. Abidi, and D. Gorsich. A simple and efficientalgorithm for part decomposition of 3-d triangulated models based on curvature analysis. IEEE ICIP, 2002.

[22] E. Zuckerberger, A. Tal, and S. Shlafman. Polyhedral surface decomposition withapplications. In Computer \& Graphics, pages 733 - 743, 2002.

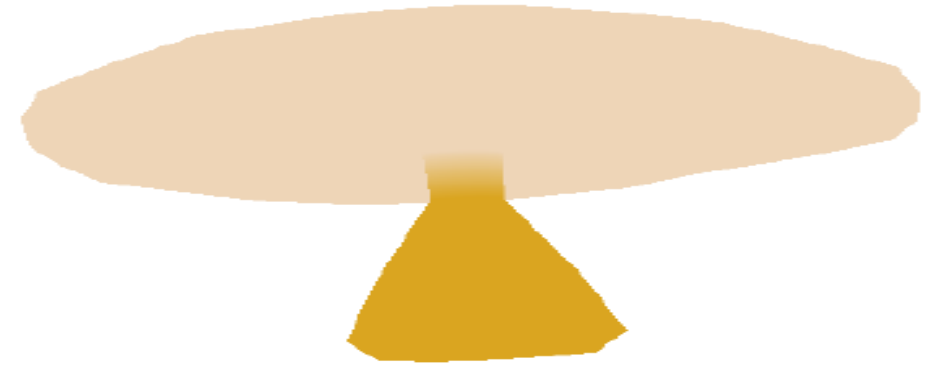

Fig. 7. Mushroom model (240 triangles) segmented to 2 regions.

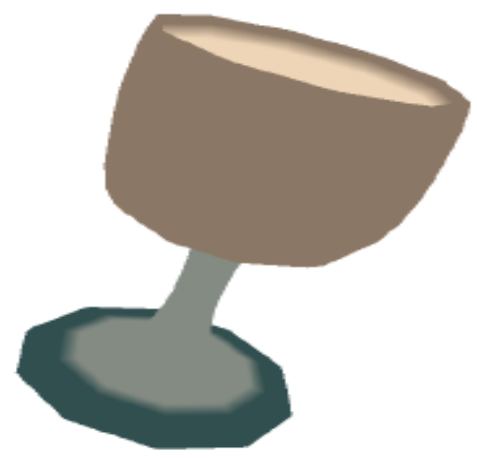

Fig. 8. Goblet model (510 triangles) segmented to 4 regions. 
International Journal of Computer Graphics \& Animation (IJCGA) Vol.5, No.2, April 2015

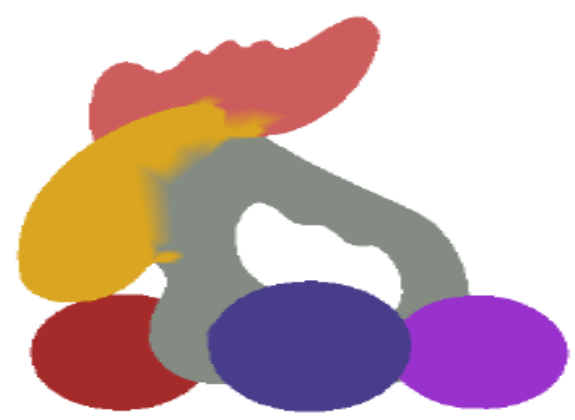

Fig. 9. Elk model (10K triangles) segmented to 7 regions.

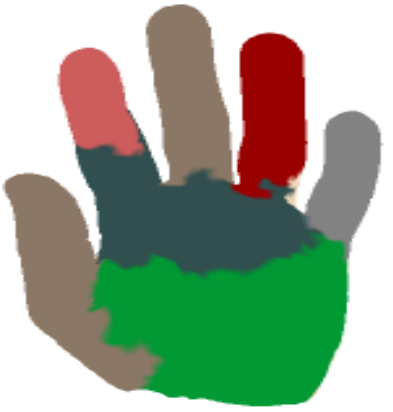

Fig. 10. Hand model (50K triangles) segmented to 8 regions.

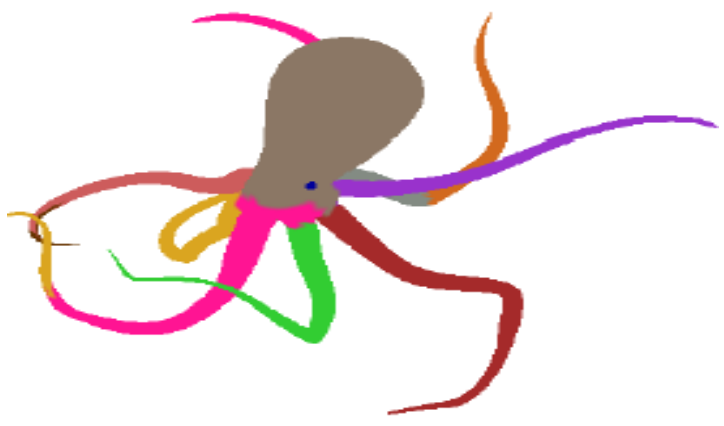

Fig. 11. Octopus model (34K triangles) segmented to 15 regions. 